\title{
ELASTIC-PLASTIC ANALYSIS OF COMBINED MODE I AND III CRACK-TIP FIELDS UNDER SMALL-SCALE YIELDING CONDITIONS
}

\author{
J. PAN $†$ and C. F. SHH $\neq$ \\ † Mechanical Engineering and Applied Mechanics, The University of Michigan, Ann Arbor, \\ MI 48109, U.S.A.; $\ddagger$ Division of Engineering, Brown University, Providence, RI 02912, U.S.A.
}

(Received 8 November 1988; in revised form 21 April 1989)

\begin{abstract}
WITHIN THE context of the small-strain approach, combined mode I and III near-tip fields of a stationary crack in an elastic-plastic solid are obtained by finite element analysis under small-scale yielding conditions. To investigate the behavior of the near-tip fields, the normalized stresses ahead of the crack tip are plotted as functions of the normalized radial distance to the tip for several combinations of prescribed mode I and III elastic $K$ fields. The angular variations of the normalized stresses at a fixed radial distance deep within the plastic zone are also plotted for several combinations of remote mode I and III elastic $K$ fields. These plots show an unmistakeable pattern : the in-plane stresses are more singular than the out-of-plane shear stresses. Over a certain distance, the near-tip in-plane stresses can be said to be more singular than $r^{-1, k n+13}$ while the near-tip out-of-plane shear stresses are less singular than $r^{-1 /(x+1)}$, where $r$ is the radial distance to the tip and $n$ is the strain hardening exponent of the material. Implications of these features as they relate to three-dimensional engineering fracture analyses are discussed.
\end{abstract}

\section{INTRODUCTION}

AsYMPToTIC plane-strain and plane-stress crack-tip stress and strain fields for powerlaw materials and perfectly plastic materials have been obtained under pure mode I and pure mode II conditions (HutCHINSON, 1968a,b; RiCE and ROSENGRen, 1968; RICE, 1968a) and under pure mode III conditions (RICE, 1968b). Cracks in typical engineering structures are generally subjected to combined mode I, II and III loading. Thus, asymptotic crack-tip fields for combined mode loadings are essential to the fracture analysis of flawed structures.

The asymptotic crack-tip stress and strain fields for power-law materials and perfectly plastic materials under combined mode I and II conditions have been presented by SHIH $(1973,1974)$. Further investigations of the combined mode I and II crack-tip fields for perfectly plastic materials can be found in NEMAT-NASSER and Obata (1984), SAKa et al. (1986) and Dong and PAN (1989a,b). PAN (1990) studied the asymptotic crack-tip fields under combined in-plane and out-of-plane loading conditions. However, the coupling of the in-plane mode and out-of-plane shear mode fields led to highly nonlincar governing differential cquations, which did not appear to be amenable to the usual separable solutions. Therefore, the governing equations 
were perturbed, and the perturbed asymptotic crack-tip fields were obtained under the assumption that either the in-plane mode or the out-of-plane shear mode is small compared to the other.

Within the small-scale yielding formulation we have obtained finite element solutions for a complete range of remotely applied combined in-plane and out-of-plane elastic $K$ fields. The in-plane and out-of-plane shear stresses are coupled through the effective stress in the plastic stress-strain relations. The out-of-plane shear stresses therefore affect the in-plane stresses in the plastically deforming region and vice versa. The purpose of our study is to examine systematically the effect of the coupling on near-tip fields. To elucidate the rather complicated nature of the near-tip fields for the complete range of combined-mode loadings, a substantial amount of numerical solutions must be presented in an appropriately normalized form. Therefore, solutions for combined mode I and III problems are discussed in this paper, and solutions for combined mode II and III problems and for combined mode I, II and III problems will be presented in subsequent papers. It may be noted that for cases where perturbation solutions for the crack-tip fields have been obtained (PAN, 1990), the perturbation solutions and the finite element solutions are in good agreement.

\section{Hutchinson-Rice-Rosengren (HRR) CRACK-tiP FieldS}

To describe the elastic-plastic behavior of the materials we consider here, we use the Ramberg-Osgood law, which is widely employed for fitting uniaxial tensile stressstrain relations :

$$
\frac{\varepsilon}{\varepsilon_{0}}=\frac{\sigma}{\sigma_{0}}+\alpha\left(\frac{\sigma}{\sigma_{0}}\right)^{n},
$$

where $\varepsilon$ is the tensile strain, $\sigma$ is the tensile stress, $\varepsilon_{0}$ and $\sigma_{0}$ are the reference strain and reference stress (we take $\varepsilon_{0}=\sigma_{0} / E$, where $E$ is Young's modulus), $\alpha$ is a material constant and $n$ is the hardening exponent. A generalization of (2.1) can be written as the sum of an elastic part $\varepsilon_{i j}^{e}$ and a volume-preserving plastic part $\varepsilon_{i j}^{p}$ :

$$
\varepsilon_{i j}=\varepsilon_{i j}^{e}+\varepsilon_{i j}^{p},
$$

where

$$
\begin{aligned}
\varepsilon_{i j}^{e} & =\frac{(1+v)}{E} s_{i j}+\frac{(1-2 v)}{3 E} \sigma_{k k} \delta_{i j}, \\
\varepsilon_{i j}^{p} & =\frac{3}{2} \alpha\left(\frac{\sigma_{e}}{\sigma_{0}}\right)^{n-1} \frac{s_{i j}}{\sigma_{0}}
\end{aligned}
$$

where $v$ is Poisson's ratio, $s_{i j}$ is the deviatoric stress and $\sigma_{e}=\left(3 s_{i j} s_{i j} / 2\right)^{1 / 2}$ is the effective stress. 


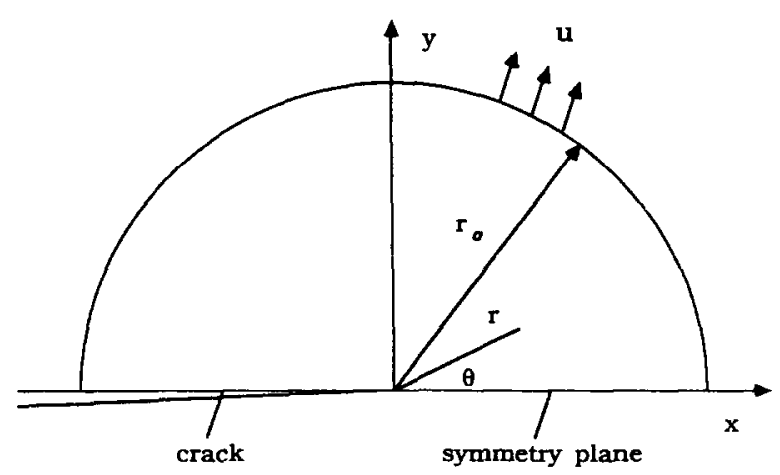

FIG. 1. A crack subjected to combined mode I and III elastic $K$ fields along the circumferential boundary.

We consider a crack in a Ramberg-Osgood solid, as shown in Fig. 1, where $r$ and $\theta$ are the polar coordinates centered at the crack tip. As $r$ approaches 0 , the linear elastic part of the strain is negligible compared to the plastic part. The asymptotic crack-tip stress, strain and displacement fields can then be expressed as (HUTCHINSON, 1968a,b; RICE and RoSENGREN, 1968; SHIH, 1973, 1974)

$$
\begin{aligned}
\sigma_{i j} & =\sigma_{0}\left(\frac{J}{\alpha \sigma_{0} \varepsilon_{0} I r}\right)^{1 /(n+1)} \tilde{\sigma}_{i j}(\theta ; n, M), \\
\varepsilon_{i j} & =\alpha \varepsilon_{0}\left(\frac{J}{\alpha \sigma_{0} \varepsilon_{0} I r}\right)^{n /(n+1)} \tilde{\varepsilon}_{i j}(\theta ; n, M), \\
u_{i}-\hat{u}_{i} & =\alpha \varepsilon_{0} r\left(\frac{J}{\alpha \sigma_{0} \varepsilon_{0} I r}\right)^{n /(n+1)} \tilde{u}_{i}(\theta ; n, M),
\end{aligned}
$$

where the dimensionless constant $I$ and the dimensionless angular functions $\tilde{\sigma}_{i j}, \tilde{\varepsilon}_{i j}$ and $\tilde{u}_{i}$ depend upon the hardening exponent $n$; the state of plane-strain, plane-stress or anti-plane deformation; and the mode parameter $M$ (mode I, mode II, mixedmode I and II, or mode III). The constants $\hat{u}_{i}$ allow for a possible rigid body motion of the crack tip itsclf. The $J$-integral (RICE, 1968a) in (2.4) represents the amplitude of the singular crack-tip stress and strain fields. Recent studies of the asymptotic crack-tip fields for power-law hardening orthotropic materials and for power-law hardening pressure-sensitive dilatant materials show the same type of functional forms as (2.4) for the asymptotic crack-tip fields (PAN and SHIH, 1986, 1988; LI and PAN, 1989a,b).

\section{Computational Model}

We consider the small-scale yielding problem depicted in Fig. 1, where the top half of a circular domain is shown. We select the upper half of the domain due to the nature of symmetry for in-plane mode I and anti-symmetry for out-of-plane mode III 
deformation. Along the remote circular boundary, displacement fields based on the mode I and mode III asymptotic crack-tip solutions for linear elastic materials are applied. The in-plane displacements $u_{i}(i=1,2)$ and the out-of-plane displacement $u_{3}$ are prescribed as

$$
u_{i}=\frac{K_{1}}{2 G}\left(\frac{r}{2 \pi}\right)^{1: 2} \tilde{u}_{i}^{1}(\theta, v)
$$

and

$$
u_{3}=\frac{K_{\mathrm{mI}}}{2 G}\left(\frac{r}{2 \pi}\right)^{i / 2} \check{u}_{3}^{\mathrm{III}}(\theta),
$$

where $G$ represents the shear modulus, and $K_{1}$ and $K_{\mathrm{II}}$ represent the far-field mode I and mode III stress intensity factors. The dimensionless functions $\tilde{u}_{i}^{\prime}(\theta, v)$ and $\tilde{u}_{3}^{\prime \prime \prime}(\theta)$ are the well-known linear elastic asymptotic displacement solutions (e.g. see KANNINEN and POPELAR, 1985).

The finite element model of the half circular domain is constructed using 9-node quadrilateral Lagrangian elements. Wedge-shaped 9-node elements are used in the immediate crack-tip region. The size of the wedge-shaped elements in the radial direction is denoted as $r_{i}$. These elements are surrounded by semi-circular strips of elements; four strips of elements span each decade of $r_{i} / r_{v}$, where $r_{v}$ denotes the radius of the half circle as shown in Fig. 1. We take $r_{i} / r_{0}=10^{-15}$ in this investigation. Therefore, 60 strips of elements, which are generated by a logarithmic scale, span the domain between $r / r_{0}=10^{-15}$ and $r / r_{0}=1$. Within each strip, the angular distance from 0 to $\pi$ is spanned by 9 elements of equal size. Therefore, the total number of elements is 549 .

The finite element formulation for this work will be discussed briefly here. Our displacement vector has an extra degree of freedom in the out-of-plane direction in addition to the two degrees of freedom of the in-plane displacements for the usual displacement-based plane-strain formulation. We used the $B$-bar method (HugHEs, 1980) to construct the strain-displacement matrix of our 9-node quadrilateral Lagrangian elements. This method alleviates the poor performance of our quadrilateral Lagrangian elements in the fully plastic range (NAGTEGAAL et al. 1974). The solution to the nonlinear system of equations is obtained by using the parameter tracking method (SHIH and NEEDLEMAN, 1984). We begin by obtaining the linear elastic solution at a load. This solution is then used as the initial estimate in the iteration for a mildly nonlinear problem with, say, $n=2$. We then use the convergent solution for the mildly nonlinear problem as the initial estimate for a more nonlinear problem. In this manner, solutions can be obtained for high-hardening to low-hardening materials. Generally speaking a convergent solution with a Euclidean error norm of about $10^{-6}$ is obtained after four to five iterations.

\section{MODE MiXITY}

To specify the far-field completely, we need two stress intensity factors, $K_{1}$ and $K_{\mathrm{II}}$. Here, we treat the angular functions $\tilde{u}_{i}^{l}(\theta, v)$ and $\tilde{u}_{3}^{111}(\theta)$ as the known quantities. 
Alternatively, we can use another set of two parameters, the $J$-integral and an elastic mixity factor, to specify the elastic far-field. The $J$-integral is related to $K_{1}$ and $K_{\mathrm{III}}$ by

$$
J=\frac{1-v^{2}}{E} K_{1}^{2}+\frac{1}{2 G} K_{\mathrm{III}}^{2} .
$$

The elastic mixity factor $M_{13}^{e}$ for our current small-scale yielding formulation is defined as

$$
M_{13}^{e}=\frac{2}{\pi} \tan ^{-1}\left[\frac{\sigma_{\theta \theta}\left(r=r_{0}, \theta=0\right)}{\sigma_{\theta z}\left(r=r_{0}, \theta=0\right)}\right]=\frac{2}{\pi} \tan ^{-1}\left[\frac{K_{1}}{K_{I I I}}\right] .
$$

According to (4.2), $M_{13}^{e}$ equals 1 for pure mode $I$ and $M_{13}^{e}$ equals 0 for pure mode III. The value of $M_{13}^{e}$ is between I and 0 for any combination of mode I and III loadings.

Within the plastic zone, the relative strength of the two modes at a distance $r$ directly ahead of the crack tip can be expressed by the plastic mixity factor $M_{13}^{p}$, defined as

$$
M_{13}^{p}(r)=\frac{2}{\pi} \tan ^{-1}\left[\frac{\sigma_{\theta \theta}(r, \theta=0)}{\sigma_{\theta z}(r, \theta=0)}\right] .
$$

Here $r$ is smaller than the smallest radial extent of the plastic zone. Thus, $M_{13}^{p}$ equals 1 for pure mode I crack-tip fields and $M_{13}^{p}$ equals 0 for pure mode III crack-tip fields. The value of $M_{13}^{p}$ ranges between 1 and 0 for combined mode near-tip fields. The plastic mixity factor $M_{13}^{p}$ is a measure of the mixture of the two modes whereas the $J$-integral is a measure of the amplitude of the near-tip fields. Important features of the combined mode I and III near-tip fields at the radial distance $r$ can be conveniently expressed by the mixity parameter and the $J$-integral.

\section{Numerical Results}

The deformation plasticity solutions for combinations of modes ranging from pure mode I to pure mode III and for hardening exponents $n$ ranging from 1 to 10 are obtained by the finite element procedure outlined in Section 3 . In these computations, we set $v=0.3$ and $\alpha=0.1$. Our finite element solutions produced the correct HRR singularities, namely $r^{-1 /(n+1)}$, for the special cases of pure mode I and pure mode III remote loadings. For the elastic problem (a test case), the numerical solutions produced the precise elastic $1 / \sqrt{r}$ singularity for various combinations of mode I and III loadings. Furthermore, for each of the convergent solutions, or $J$ values, as calculated by the domain integral method (Li et al., 1985; SHIH et al., 1986; MORAN and SHIH, 1987) for each of the semi-circular strips, differed by less than one per cent from the prescribed value as determined by (4.1). The path-independence of the computed $J$ values and the excellent agreement with (4.1) attest to the quality of four finite element solutions.

By the process of parameter tracking, we have obtained solutions for the full range 
of $n$ values $(1 \leqslant n \leqslant 10)$ and mixtures of mode I and III. To keep the paper within a reasonable length, only solutions for $n=3$ and 10 and for remote displacement boundary conditions corresponding to five mixities, namely, $M_{13}^{c}=1,0.83,0.5,0.17$ and 0 , are presented in Figs 2-6. These five cases represent the pure mode I, $K_{\mathrm{III}} / K_{\mathrm{I}}=0.27, K_{\mathrm{III}} / K_{\mathrm{I}}=1, K_{1} / K_{\mathrm{III}}=0.27$ and the pure mode III cases.

To examine the dependence of the near-tip stresses on the radial distance $r$ (at fixed $\theta$ ), we plot the normalized stresses (in the $x, y$ and $z$ coordinates) ahead of the crack tip as functions of the normalized radial distance to the tip using a $\log -\log$ scale. The numerically determined stresses are normalized by the HRR singularity such that $\bar{\sigma}_{i j}=\sigma_{i /}\left[\sigma_{0}\left(J / \alpha \sigma_{0} \varepsilon_{0} r\right)^{1:(n+1)}\right]$. The radial distance is normalized by the length of the plastic zone, $r_{p}$, ahead of the crack tip. The normalized stresses plotted in the figures are based on the actual computed values in the $x, y$ and $z$ coordinates along the central column (at $\theta=10^{\circ}$ ) of the Gauss points in the first wedge strip of the elements immediately above the symmetry plane. Comparisons of the solutions at different $\theta \mathrm{s}$ as functions of the normalized radial distance deep within the plastic zone indicate that the nature of the singularity is virtually the same. In order to investigate the mathematical structure of the near-tip fields and to reveal the similarities (if any) between the combined-mode near-tip fields and the pure mode I and mode III HRR fields, it is necessary to examine fields that are well below those that are physically relevant. For example, we have plotted the radial variations of the normalized stresses for distances as small as $r / r_{p} \approx 10^{-13}$ to $10^{-14}$ (depending upon the mixity of the modes). In applying these solutions to the analysis of fracture under combined-mode loading, fields within such distances would obviously be disregarded.

To examine the angular variations of the near-tip stress fields, we plot the normalized stresses $\bar{\sigma}_{i, j}$ (in the $r, \theta$ and $z$ coordinates) as functions of $\theta$ at a radial distance of $r / r_{p} \approx 10^{-3}$. The normalized stresses plotted in the figures are based on the computed values in the $x, y$ and $z$ coordinates along the central row of the Gauss points in the semi-circular strip of the elements at about $r / r_{n} \approx 10^{-3}$.

As mentioned previously, the first ring consists of wedge-shaped elements where one edge of every element is collapsed onto a point which is the crack tip. In most of our numerical studies, the three nodes at the edge under discussion are tied together, and every node at the crack tip displaces together as a single point. We have also used a wedge element where the three nodes at the crack tip can displace independently. Displacement gradients associated with the latter element contain terms of order $(1 / r)$. For the purpose of sorting out the radial dependence and the angular dependence of the stresses we used stresses computed from elements which are located at distances which are several times larger than the linear dimension of the wedge elements. Thus the type of crack-tip elements has little or no effect on the numerical results that are presented. In addition, for each problem analysed, the magnitude of the far-field displacement field is chosen so that the maximum extent of the plastic zone size (as a function of $\theta$ ) is about 10 per cent of $r_{0}$ at the most. Thus, the small-scale yielding conditions are not violated and the stresses deep within the plastic zone can be investigated. We have carried out computations which resulted in values of $r_{p} / r_{0}$ ranging from 0.1 to 0.001 .

Solutions for each of the five cases in the order of departure from mode I are presented below. 
(a)
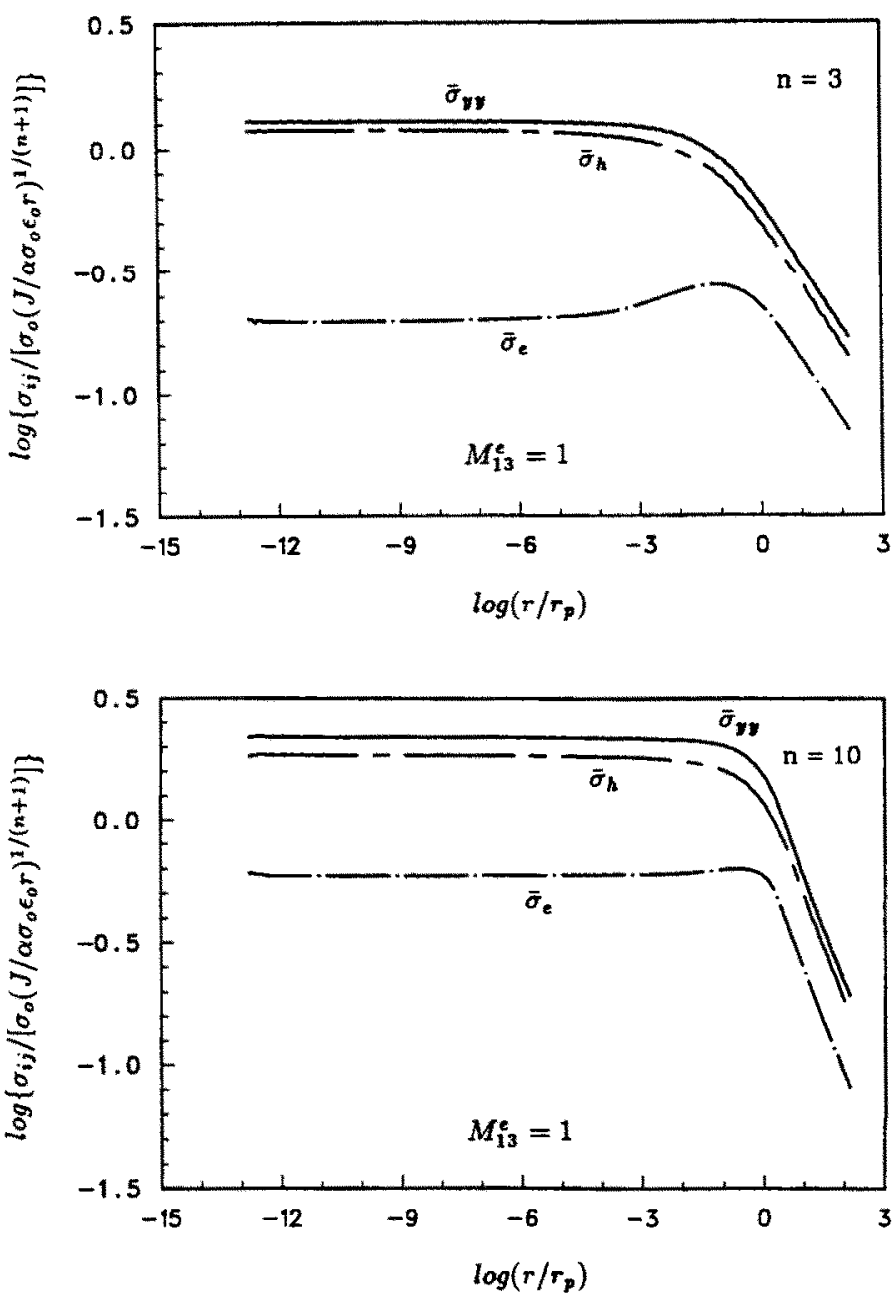

Fig. 2. Case $1\left(M_{13}^{\varepsilon}=1\right.$, pure mode $\left.I\right)$ : the normalized stresses $\bar{\sigma}_{i j}$ at $\theta=10^{\circ}$ as functions of $r / r_{p}$ plotted in a $\log -\log$ scale for (a) $n=3$ and (b) $n=10$; the normalized stresses $\bar{\sigma}_{i j}$ at $r / r_{p} \approx 10^{-3}$ plotted as functions of $\theta$ for (c) $n=3$ and (d) $n=10$.

\section{Case 1: $\mathbf{M}_{13}^{\mathrm{e}}=1$ (pure mode $I$ )}

Figure 2 ( $a$ and $b$ ) shows the normalized opening stress $\bar{\sigma}_{y y}$, the normalized hydrostatic stress $\bar{\sigma}_{h}$ and the normalized effective stress $\bar{\sigma}_{e}$ at $\theta=10^{\circ}$ as functions of the normalized radial distance $r / r_{p}$ in a $\log -\log$ scale for $n=3$ and 10 , respectively. It may be noted that for cases 1-5 shown in Figs 2-6, outside of the plastic zone $\left(\log \left(r / r_{p}\right)>0\right)$ the variation of the stresses with the radial distance is in agreement with the elastic singularity. Within the plastic zone, the stresses begin to level off at about $r / r_{p} \approx 10^{-2}$ for $n=3$ and at about $r / r_{p} \approx 10^{-1}$ for $n=10$. Since the numerically 
(c)
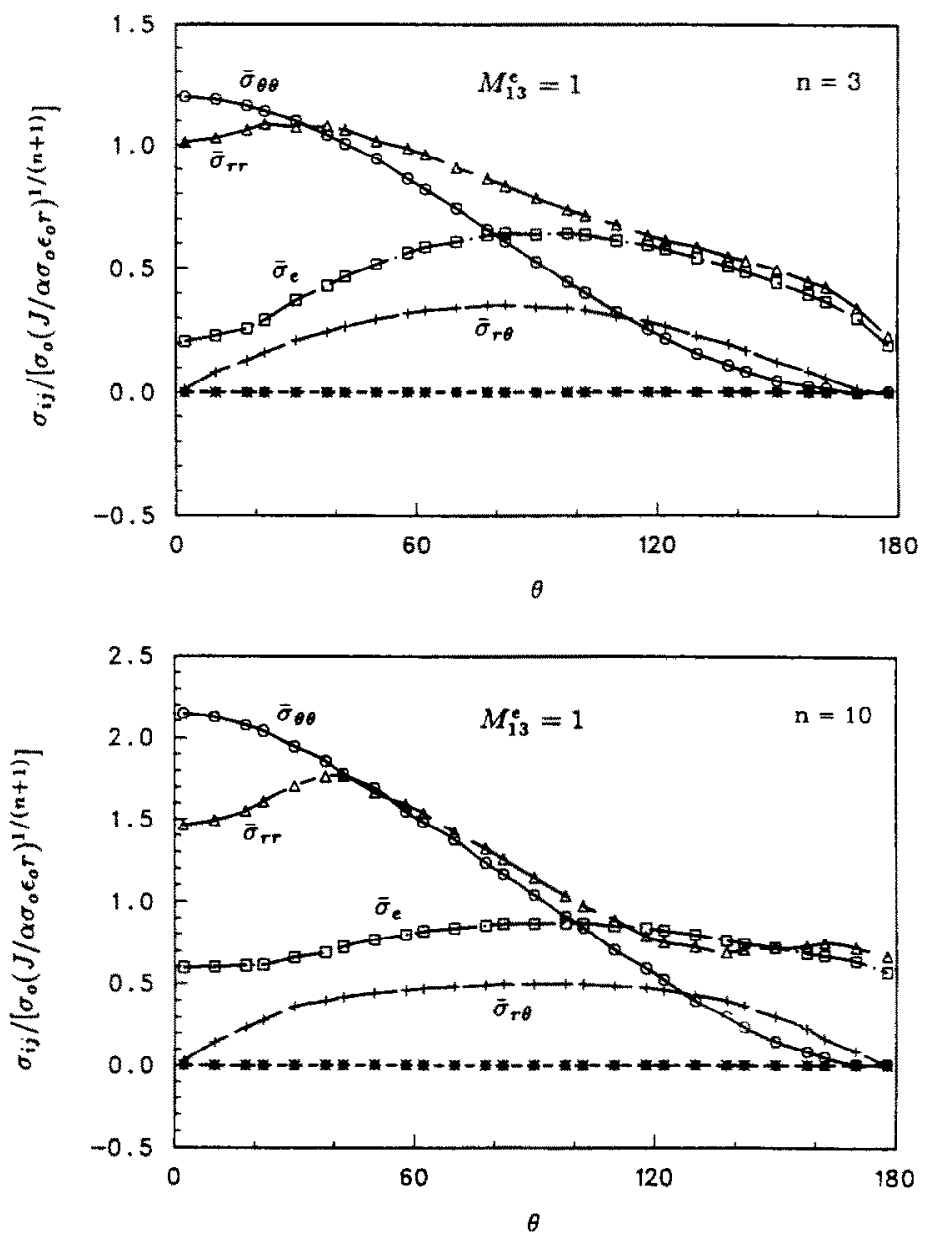

FIG. $2 c$ and $d$.

determined stresses are normalized by the HRR singularity, the levelling off means that the radial variation of the stresses is in accord with the HRR singularity. Indeed, the magnitudes of the computed stresses are equal to those of the HRR singularity; furthermore, these computed magnitudes appropriate to the HRR singularity are achieved much more rapidly for the lower hardening $(n=10)$ material. Although we have plotted only the stresses $\bar{\sigma}_{v y}, \bar{\sigma}_{h}$ and $\bar{\sigma}_{e}$, the other three stresses $\bar{\sigma}_{v y}, \bar{\sigma}_{r v}$ and $\bar{\sigma}_{y z}$ show the same trends. In the case of the $n=10$ material, which is a representative of the hardening characteristics of typical structural steels, the HRR fields are good approximations of the actual stresses and strains for $r / r_{p} \leqslant 10^{-1}$ (note that $r / r_{p}=10^{-1}$ is a physically relevant distance).

Figure 2 (c and d) shows the normalized stresses $\bar{\sigma}_{i j}$ at about $r / r_{p} \approx 10^{-3}$ as functions of $\theta$ for $n=3$ and $n=10$, respectively. The angular functions of the normalized stresses closely resemble the HRR asymptotic solutions (for example, see the solutions 
(a)

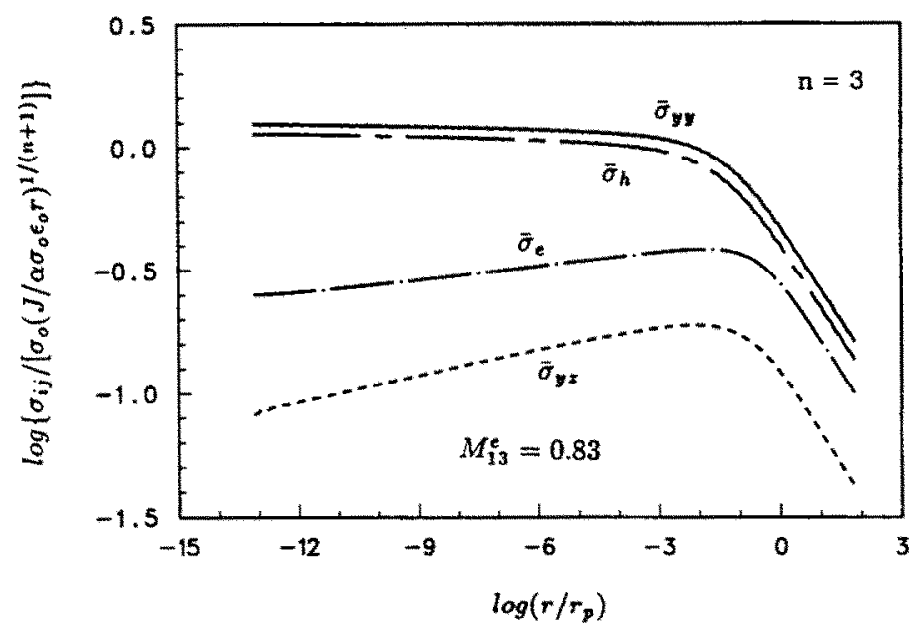

(b)

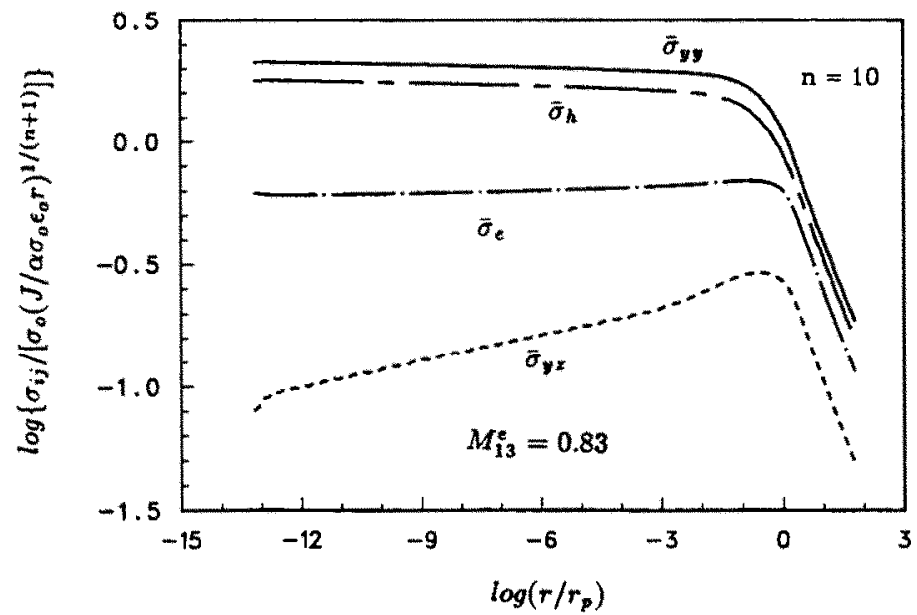

F1G. 3. Case $2\left(M_{i},=0.83, K_{\mathrm{n}} / K_{\mathrm{I}}=0.27\right)$ : the normalized stresses $\bar{\sigma}_{i f}$ at $\theta=10^{\circ}$ as functions of $r / r_{\phi}$ plotted in a $\log -\log$ scale for (a) $n=3$ and (b) $n=10$; the normalized stresses $\bar{\sigma}_{i j}$ at $r i r_{p} \approx 10^{-3}$ plotted as functions of $\theta$ for (c) $n=3$ and (d) $n=10$.

plotted in PAN, 1990). The solutions for this case serve to check the accuracy of our finite element solutions.

Case 2: $\mathrm{M}_{13}^{\mathrm{e}}=0.83\left(\mathrm{~K}_{\mathrm{III}} / \mathrm{K}_{1}=0.27\right)$

Figure 3 ( $\mathrm{a}$ and $\mathrm{b}$ ) shows the normalized opening stress $\bar{\sigma}_{y y}$, the normalized hydrostatic stress $\tilde{\sigma}_{h}$, the normalized effective stress $\tilde{\sigma}_{e}$, and the normalized out-of-plane shear stress $\bar{\sigma}_{y z}$ at $\theta=10^{\circ}$ as functions of $r / r_{p}$ in a $\log -\log$ scale for $n=3$ and 10 , respectively. As shown in the figures, well within the plastic zone $\bar{\sigma}_{y z}$ and $\bar{\sigma}_{e}$ decrease 
(c)

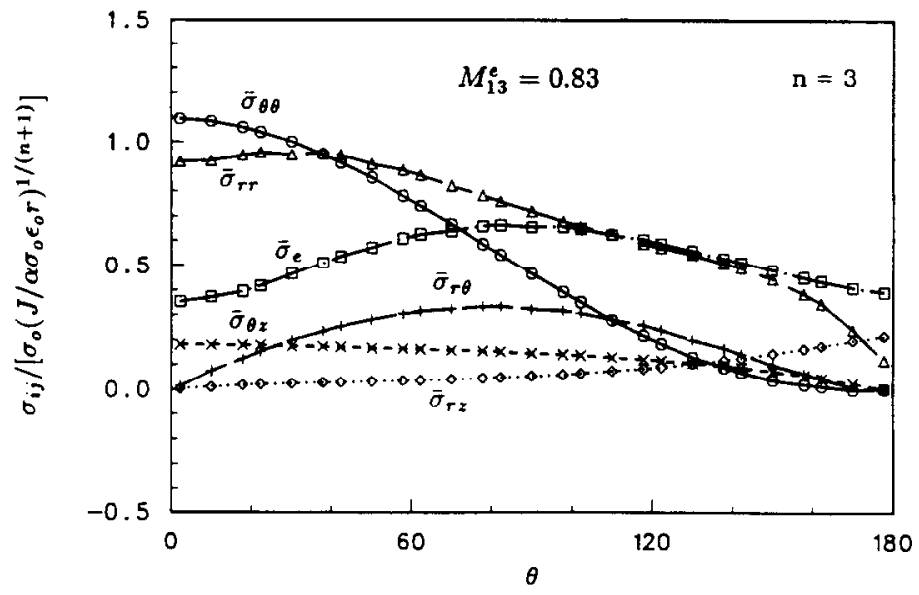

(d)

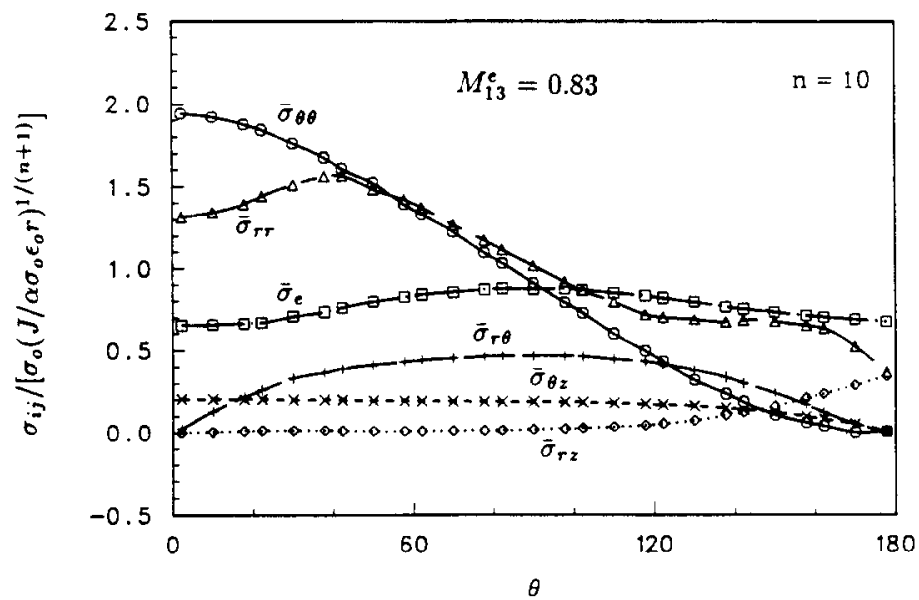

FIG. $3 \mathrm{c}$ and $\mathrm{d}$.

whereas $\bar{\sigma}_{y y}$ and $\bar{\sigma}_{h}$ increase as $r / r_{p}$ decreases. Note that for this case (and for cases 3 and 4) the two other in-plane stresses, $\bar{\sigma}_{x x}$ and $\bar{\sigma}_{x x}$, show the same trend as that of $\bar{\sigma}_{y y}$, and the other out-of-plane shear stress $\bar{\sigma}_{x z}$ shows the same trend as that of $\bar{\sigma}_{y z}$. This suggests that well within the plastic zone and well within the range of $r / r_{p}$ indicated in the figures, the in-plane stresses are more singular than the out-of-plane shear stresses. However, $\bar{\sigma}_{y y}, \bar{\sigma}_{h}$ and $\bar{\sigma}_{e}$ have the tendency to level off, which suggests

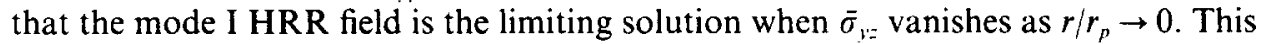
trend is more clear in Fig. 3(b) for $n=10$. Since the contribution of the out-of-plane shear stresses (relative to the in-plane stresses) to the effective stress vanishes in the limit as $r / r_{p} \rightarrow 0$, the singularity of the in-plane stresses, $\bar{\sigma}_{h}$ and $\bar{\sigma}_{c}$ must asymptotically approach the HRR singularity.

As shown in the figures, the weakening of the out-of-plane shear stresses as $r / r_{p}$ decreases is more pronounced for low-hardening materials (with large $n$ ). From the figures, the differences of the singularity of $\bar{\sigma}_{r z}$ in $r$ from the HRR singularity are 
approximately estimated as 0.03 for $n=3$ and 0.04 for $n=10$ averaged over ten decades of $r / r_{p}$. These values agree with the value of 0.0462 for $n=3$ and 0.0472 for $n=10$ from the perturbation analysis of the asymptotic behavior of the crack-tip fields (PAN, 1990).

Figure 3 (c and d) shows the normalized stresses $\bar{\sigma}_{i j}$ at about $r / r_{n} \approx 10^{-3}$ as functions of $\theta$ for $n=3$ and 10 , respectively. The angular variations of the out-of-plane stresses $\bar{\sigma}_{r z}$ and $\bar{\sigma}_{\theta z}$ differ from those of the pure mode III case (Fig. $6 \mathrm{c}$ and d). The characteristics of these functions, however, agree well with those of the asymptotic analysis of PAN (1990). The in-plane stresses nearly preserve the angular functional forms of the pure mode I HRR fields (Fig. $2 \mathrm{c}$ and d) except for a slight drop of $\vec{\sigma}_{r r}$ near 180 due to the large value of $\bar{\sigma}_{r:}$.

The elastic mixity factor $M_{13}^{e}$ is about $0.83\left(\sigma_{\theta z}(\theta=0) / \sigma_{\theta \theta}(\theta=0)=0.27\right)$. As $r / r_{p}$ decreases from unity, the plastic mixity factor $M_{13}^{p}$ increases. At about $r / r_{p} \approx 10^{-3}$, the plastic mixity factor $M_{13}^{p}$ is 0.89 for $n=3$ and 0.93 for $n=10$ $\left(\sigma_{\theta z}(\theta=0) / \sigma_{\theta \theta}(\theta=0)=0.17\right.$ for $n=3$ and 0.11 for $\left.n=10\right)$. At a very small distance, $r / r_{p} \approx 10^{-13}$, the plastic mixity factor $M_{13}^{p}$ increases to 0.96 for $n=3$ and 0.98 for $n=10\left(\sigma_{\theta z}(\theta=0) / \sigma_{\theta \theta}(\theta=0)=0.06\right.$ for $n=3$ and 0.03 for $\left.n=10\right)$. This demonstrates that the near-tip fields approach the pure mode I field as $r / r_{p}$ decreases, and this behavior is more pronounced for low-hardening materials (with large $n$ ).

\section{Case 3: $\mathrm{M}_{13}^{\mathrm{c}}=0.5\left(\mathrm{~K}_{111} / \mathrm{K}_{1}=1\right)$}

Figure 4 (a and b) shows the normalized stresses $\bar{\sigma}_{y x}, \bar{\sigma}_{b}, \bar{\sigma}_{\mathrm{e}}$ and $\bar{\sigma}_{x=}$ at $\theta=10^{\circ}$ as functions of $r / r_{p}$ in a $\log -\log$ scale for $n=3$ and 10 , respectively. As shown in the figures, well within the plastic zone $\bar{\sigma}_{y z}$ and $\bar{\sigma}_{e}$ decrease whereas $\bar{\sigma}_{y v}$ and $\bar{\sigma}_{h}$ increase as $r / r_{p}$ decreases. These stress plots indicate that well within the plastic zone, the singularity of the in-plane stresses is slightly stronger than that of the out-of-plane shear stresses. For the $n=3$ case, which is shown in Fig. $4(a), \bar{\sigma}_{y r}$ and $\bar{\sigma}_{h}$ tend to level off to the HRR singularity while $\bar{\sigma}_{y z}$ and $\bar{\sigma}_{c}$ decrease as $r / r_{p}$ decreases.

The trends noted above are more clearly developed for the $n=10$ case, which is shown in Fig. 4(b). Well within the plastic zone, the normalized stresses $\bar{\sigma}_{y y}, \bar{\sigma}_{h}$ and $\bar{\sigma}_{e}$ have nearly leveled off and can be said to be in accord with the HRR singularity. The vanishing of $\bar{\sigma}_{r=}$ as $r / r_{p} \rightarrow 0$ is a sufficient condition for the development of the HRR singularity. A comparison of Fig. 4 ( $a$ and $b$ ) shows that the weakening of the out-of-plane shear stresses as $r / r_{p}$ decreases is more pronounced for low-hardening materials (with large $n$ ). The trends of the numerical solutions (for $n=3$ and $n=10$ ) strongly suggest that asymptotically, as $r / r_{p} \rightarrow 0$, the in-plane stresses, $\bar{\sigma}_{k}$ and $\bar{\sigma}_{k}$ approach those corresponding to the mode I HRR singularity.

In Fig. 4(b) (for $n=10$ ), where this behavior is more readily apparent, the difference of the singularity of $\tilde{\sigma}_{y z}$ from the HRR singularity averaged over ten decades of $r / r_{p}$ is estimated at 0.04 . This value agrees with the value of 0.0472 for $n=10$ from the perturbation analysis of the asymptotic behavior of the crack-tip fields (PAN, 1990). For $n=3$ the contribution of the out-of-plane shear stresses to the effective stresses compared to that of the in-plane stresses is not small for the range of $r / r_{p}$ considered. Therefore, the perturbation results of PAN (1990) cannot be used to estimate the singularity difference of the out-of-plane shear stresses from the HRR singularity. 
(a)

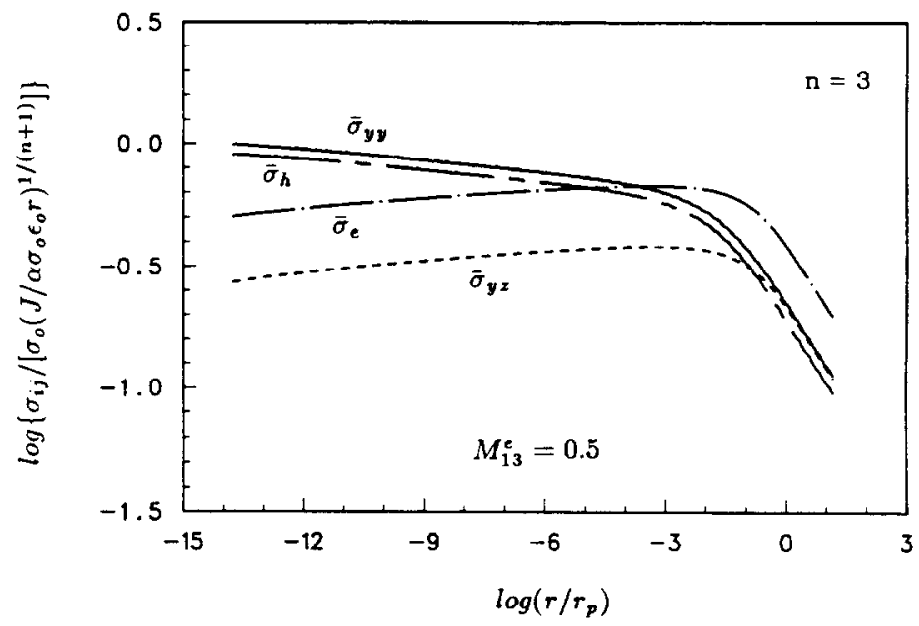

(b)

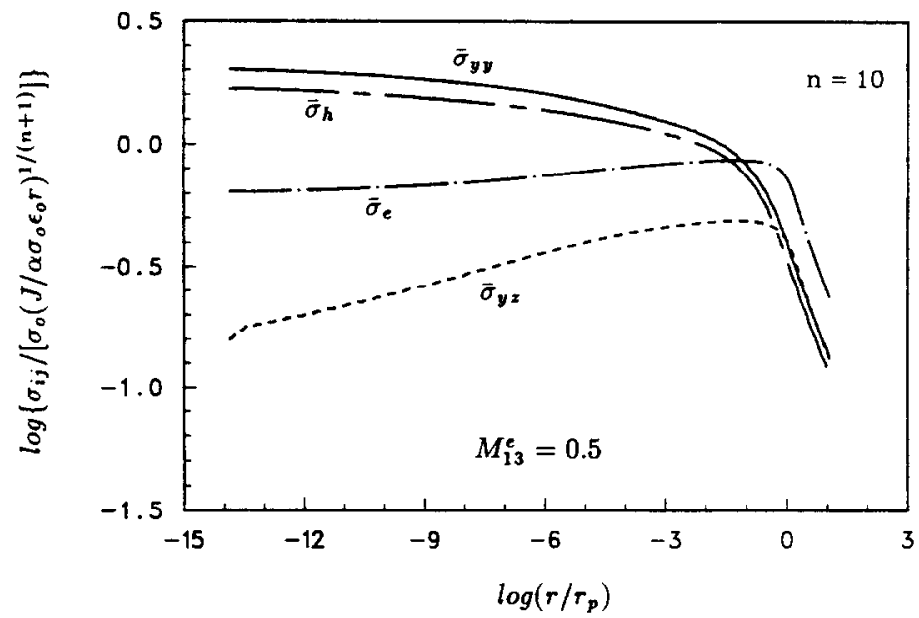

Fig. 4. Case $3\left(M_{13}^{e}=0.5, K_{\mathrm{III}} / K_{\mathrm{I}}=1\right)$ : the normalized stresses $\bar{\sigma}_{i j}$ at $\theta=10$ as functions of $r / r_{p}$ plotted in a $\log -\log$ scale for (a) $n=3$ and (b) $n=10$; the normalized stresses $\bar{\sigma}_{i j}$ at $r i r_{n} \approx 10^{-3}$ plotted as functions of $\theta$ for (c) $n=3$ and (d) $n=10$.

Figure 4 (c and d) shows the normalized stresses $\bar{\sigma}_{i j}$ at about $r / r_{p} \approx 10^{-3}$ as functions of $\theta$ for $n=3$ and 10 , respectively. The angular functions of the out-of-plane shear stresses $\bar{\sigma}_{r z}$ and $\bar{\sigma}_{\theta z}$ for $n=3$ and 10 resemble those of the pure mode III case (Fig. $6 \mathrm{c}$ and d). In these figures, the magnitude of the in-plane stresses is larger than or comparable to that of the out-of-plane shear stresses, but the contribution of the inplane stresses to the effective stress is actually smaller than that of the out-of-plane shear stresses. Therefore, the angular functions of the in-plane stresses shown in Fig. 4 ( $c$ and d) possess some of the characteristics of the perturbed mode I solutions (PAN, 1990).

The clastic mixity factor $M_{13}^{e}$ is $0.5\left(\sigma_{\theta z}(\theta=0) / \sigma_{\theta \theta}(\theta=0)=1\right)$. As $r / r_{p}$ decreases from unity, the plastic mixity factor $M_{13}^{p_{13}}$ increases. At about $r / r_{p} \approx 10^{-3}$, the plastic 
(c)
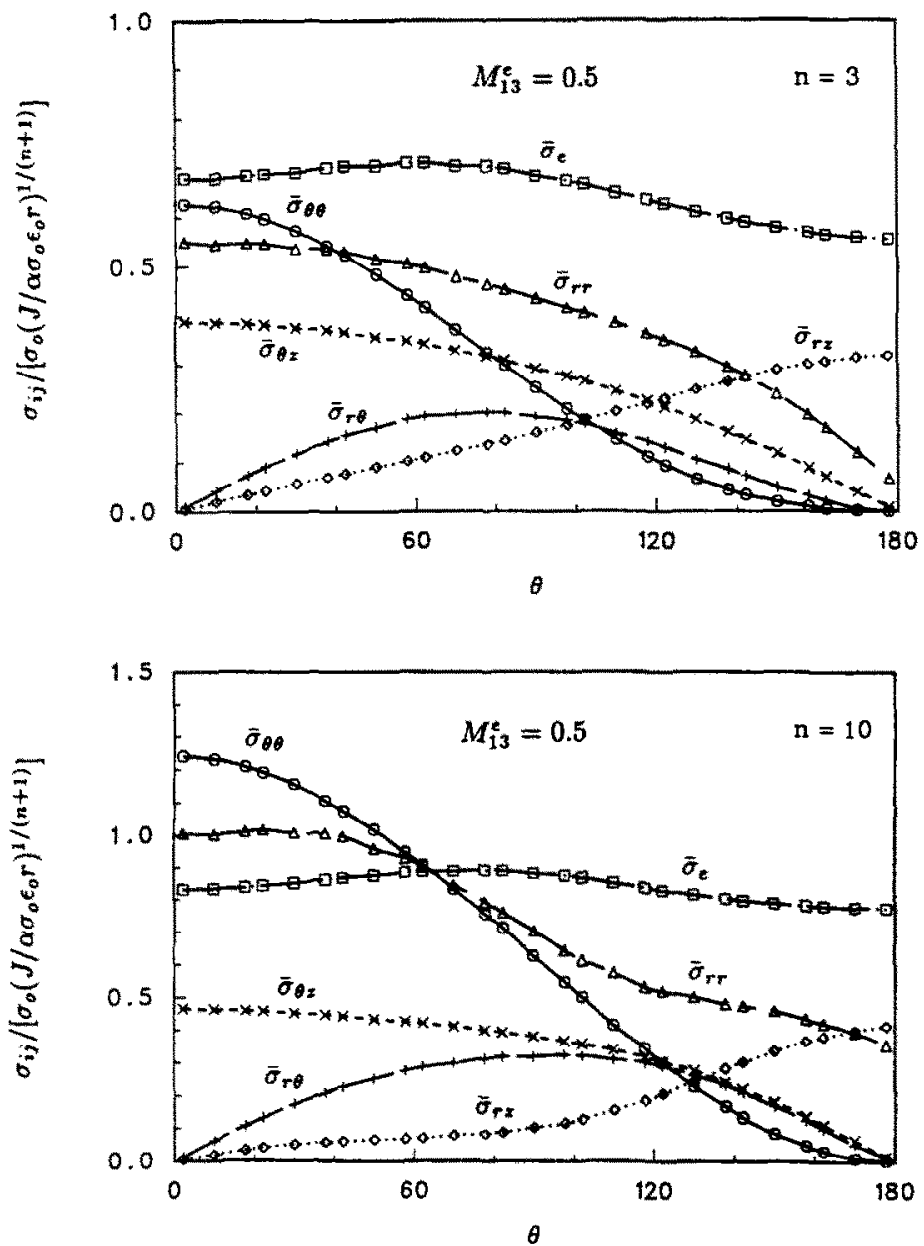

FIG. $4 \mathrm{c}$ and $\mathrm{d}$.

mixity factor $M_{13}^{p}$ is 0.65 for $n=3$ and 0.76 for $n=10\left(\sigma_{\theta z}(\theta=0) / \sigma_{\theta \theta}(\theta=0)=0.62\right.$ for $n=3$ and 0.38 for $n=10$ ). At a very small distance, $r / r_{p} \approx 10^{-14}$, the plastic mixity factor $M_{13}^{p}$ increases to 0.81 for $n=3$ and 0.95 for $n=10\left(\sigma_{\theta z}(\theta=0) /\right.$ $\sigma_{\theta \theta}(\theta=0)=0.3$ for $n=3$ and 0.08 for $n=10$ ). This demonstrates again that the near-tip fields approach the pure mode I field as $r / r_{p}$ decreases, and this effect is more pronounced for low-hardening materials (with large $n$ ).

Case 4: $\mathrm{M}_{13}^{\mathrm{c}}=0.17\left(\mathrm{~K}_{1} / \mathrm{K}_{\mathrm{III}}=0.27\right)$

Figure $5\left(\mathrm{a}\right.$ and b) shows the normalized stresses $\bar{\sigma}_{y y}, \bar{\sigma}_{h}, \bar{\sigma}_{e}$ and $\bar{\sigma}_{y z}$ at $\theta=10^{\circ}$ as functions of $r / r_{p}$ in a $\log -\log$ scale for $n=3$ and 10 , respectively. Within the plastic zone for $n=3$ (see Fig. 5a), $\bar{\sigma}_{y z}$ and $\bar{\sigma}_{e}$ level off rather quickly (the stresses are well approximated by the HRR singularity in this interval) and then begin to decrease 
(a)
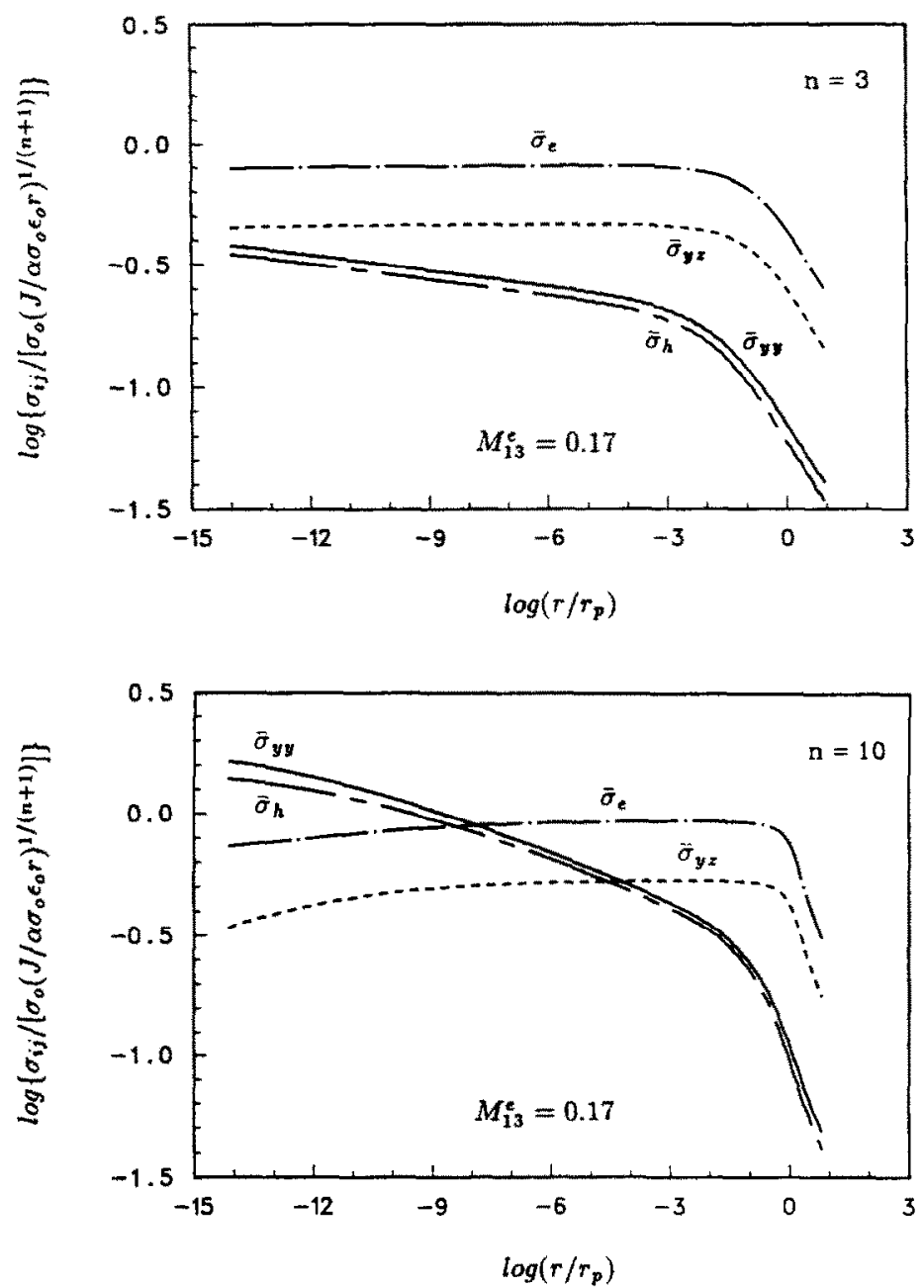

FIG. 5. Case $4\left(M_{13}^{e}=0.17, K_{\mathrm{I}} / K_{\mathrm{III}}=0.27\right)$ : the normalized stresses $\bar{\sigma}_{i j}$ at $\theta=10$ as functions of $r / r_{p}$ plotted in a $\log \log$ scale for (a) $n=3$ and (b) $n=10$; the normalized stresses $\bar{\sigma}_{i j}$ at $r / r_{p} \approx 10^{-3}$ plotted as functions of $\theta$ for (c) $n=3$ and (d) $n=10$.

slightly. In contrast, the in-plane stress $\bar{\sigma}_{y y}$ and $\bar{\sigma}_{h}$ increase monotonically as $r / r_{p}$ decreases. In Fig. 5(b), which is for $n=10, \tilde{\sigma}_{1=}$ and $\bar{\sigma}_{c}$ quickly level off and then begin to decrease. The in-plane stress $\bar{\sigma}_{y y}$ and $\bar{\sigma}_{h}$ increase and then show the tendency to level off as $r / r_{p}$ decreases. The lower limit of the range of $r / r_{p}$ shown in Fig. 5(a) for $n=3$ is perhaps not small enough to reveal the unmistakeable trend shown in Fig. 5 (b) for $n=10$.

The numerical solutions support the observation made in connection with the preceding cases: well within the plastic zone the in-plane stresses are more singular than the out-of-plane shear stresses. Note that $\bar{\sigma}_{w y}, \bar{\sigma}_{h}$ and $\bar{\sigma}_{i}$ tend to level off at values 
(c)
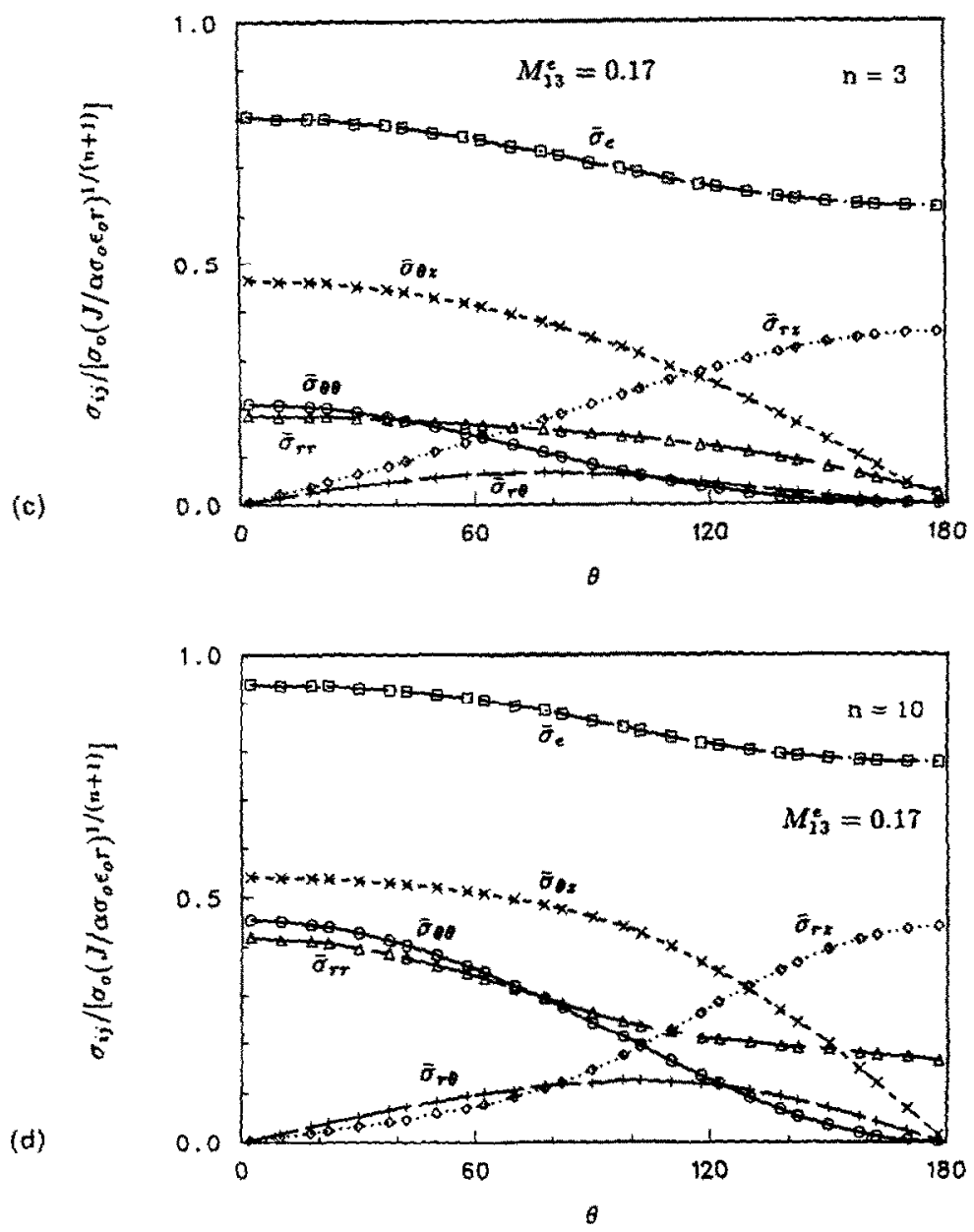

Fig. Sc and d.

appropriate to the HRR singularity as $\bar{\sigma}_{y=}$ decreases with the decrease of $r / r_{r}$. This tendency is more pronounced for low-hardening materials, as shown in Fig. $5(\mathrm{~b})$ for $n=10$. This trend suggests that as $r / r_{p} \rightarrow 0$, the relative contribution of the out-ofplane shear stresses to the effective stress vanishes, and the in-plane stresses, $\bar{\sigma}_{h}$ and $\bar{\sigma}_{e}$ must asymptotically approach the HRR singularity. From the figures, the differences of the singularity of $\tilde{\sigma}_{,}$in $r$ from the HRR singularity are approximately estimated at 0.03 for $n=3$ and 0.06 for $n=10$ averaged over ten decades of $r / r_{p}$. These values agree with the values of 0.0263 for $n=3$ and 0.0709 for $n=10$ from the perturbation analysis of the asymptotic crack-tip fields (PAN, 1990).

For both $n=3$ and $n=10$, the magnitude of the in-plane stresses becomes large compared to that of the out-of-plane shear stresses as $r / r_{p}$ decreases. For $n=3$, the contribution of the in-plane stresses to the effective stress is smaller than that of the out-of-plane shear stresses for the range of $r / r_{p}$ considered here. Therefore, the results 
of the singularity difference from the HRR singularity of the finite element computations agree well with the results of the perturbation analysis of PAN (1990). For $n=10$, the contribution of the in-plane stresses to the effective stress is still smaller than that of the out-of-plane shear stresses within most of the range of $r / r_{p}$ considered here. Close to the lower limit of $r / r_{p}$ shown in the figure the contribution of the inplane stresses to the effective stress becomes comparable to that of the out-of-plane shear stresses. However, the results of the singularity difference from the HRR singularity of the finite element computations agree well with the results of the perturbation analysis of PAN (1990).

The results that have been presented show that the near-tip plasticity has the effect of increasing the magnitude of the in-plane stresses relative to the out-of-plane shear stresses. This effect is clearly revealed by the curves in Fig. 5(b). Here a small amount of remote mode I loading added to a dominant mode III loading leads to in-plane stresses and hydrostatic stress that are much larger than the out-of-plane shear stresses as $r / r_{p}$ decreases. The rather high hydrostatic stresses in the near-tip region may provide an explanation for the experimental results obtained under combined mode I and III loading conditions by MigLin et al. (1984). The surfaces of their fractured test specimens showed the ductile processes of void initiation, growth. and coalescence, which are usually associated with high hydrostatic stresses.

Figure 5 (c and d) shows the normalized stresses $\tilde{\sigma}_{i j}$ at about $r / r_{p} \approx 10^{-3}$ as functions of $\theta$ for $n=3$ and 10 , respectively. In these figures, the contribution of the in-plane stresses to the effective stress is small compared to that of the out-of-plane shear stresses. The angular functions of the in-plane stresses, therefore, agree well with the perturbed mode I solutions of PAN (1990). The angular functions of the out-of-plane shear stresses $\bar{\sigma}_{r z}$ and $\bar{\sigma}_{\theta z}$ resemble those of the pure mode III case (Fig. $6 \mathrm{c}$ and d).

The mixity factor $M_{13}^{e}$ is about $0.17\left(\sigma_{\theta \theta}(\theta=0) / \sigma_{\theta z}(\theta=0)=0.27\right)$ at the remotc elastic far-field. As $r / r_{p}$ decreases from unity, the plastic mixity factor $M_{13}^{p}$ increases. At about $r / r_{p} \approx 10^{-3}$, the plastic mixity factor $M_{13}^{p}$ is 0.27 for $n=3$ and 0.45 for $n=10\left(\sigma_{\theta \theta}(\theta=0) / \sigma_{\theta z}(\theta=0)=0.45\right.$ for $n=3$ and 0.84 for $\left.n=10\right)$. At a very small distance, $r / r_{p} \approx 10^{-14}$, the plastic mixity factor $M_{13}^{p}$ increases to 0.43 for $n=3$ and 0.85 for $n=10\left(\sigma_{\theta \theta}(\theta=0) / \sigma_{\theta z}(\theta=0)=0.8\right.$ for $n=3$ and 4.3 for $\left.n=10\right)$. This demonstrates that the near-tip fields approach the pure mode I field as $r / r_{p}$ decreases, and this effect is more pronounced for low-hardening materials (with large $n$ ). As shown in Fig. 5(b) for $n=10$, the dominant mode switches from mode III at $r / r_{p}=1$ to mode $I$ at about $r / r_{p} \approx 10^{-4}$.

\section{Case $5: \mathrm{M}_{13}^{\mathrm{e}}=0$ (pure mode III)}

Figure 6 (a and b) shows the normalized stresses $\bar{\sigma}_{y=}$ and $\bar{\sigma}_{e}$ at $\theta=10^{\circ}$ as functions of $r / r_{p}$ in a $\log -\log$ scale for $n=3$ and 10 , respectively. As shown in the figures, within the plastic zone the stresses begin to level off at $r / r_{n} \approx 10^{-2}$ for $n=3$ and at about $r / r_{p} \approx 10^{-1}$ for $n=10$. In other words, the HRR singularity dominates over these distances. Although we have plotted the behavior of the two representative stresses, the trend of the other shear stress $\bar{\sigma}_{x z}$ is identical. It may be noted that for lowhardening materials (with large $n$ ), the HRR singularity is approached much more rapidly. 
(a)

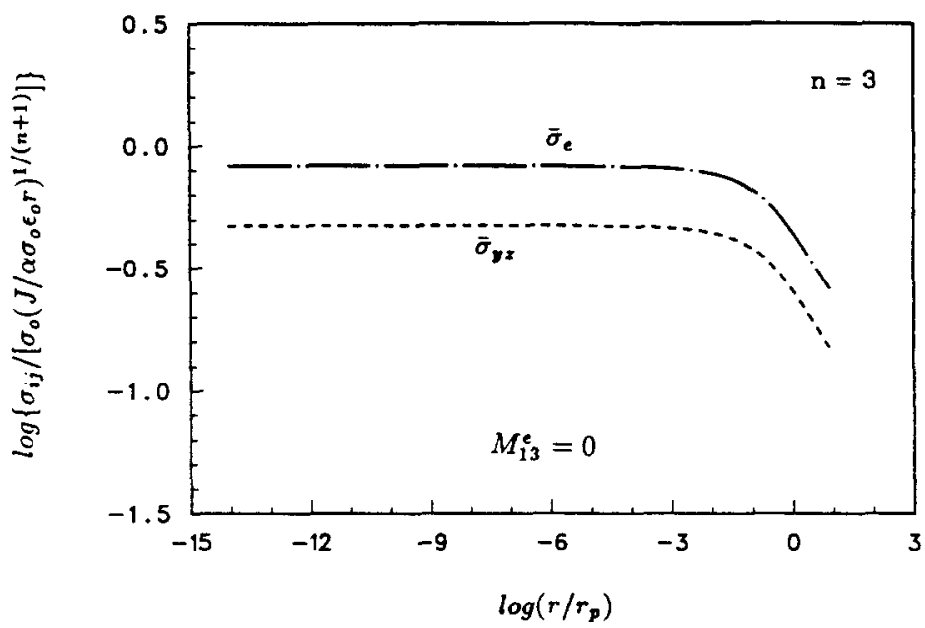

(b)

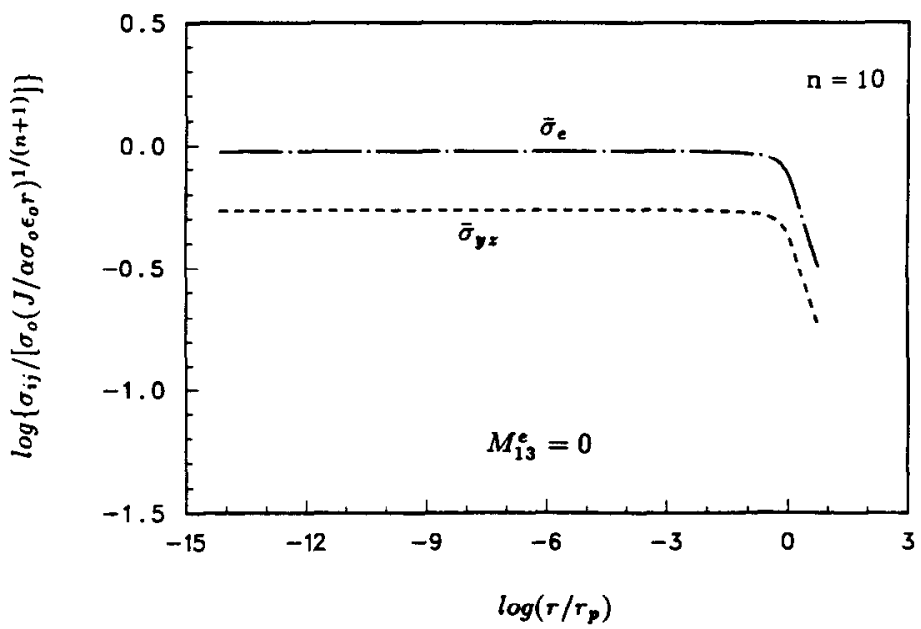

Fig. 6. Case 5 ( $M_{13}^{e}=0$, pure mode III) : the normalized stresses $\bar{\sigma}_{i j}$ at $\theta=10^{\circ}$ as functions of $r / r_{p}$ plotted in a $\log -\log$ scale for (a) $n=3$ and (b) $n=10$; the normalized stresses $\tilde{\sigma}_{i j}$ at $r / r_{p} \approx 10^{-3}$ plotted as functions of $\theta$ for (c) $n=3$ and (d) $n=10$.

Figure 6 ( $\mathrm{c}$ and $\mathrm{d}$ ) shows the normalized stresses $\bar{\sigma}_{i j}$ at about $r / r_{p} \approx 10^{-3}$ as functions of $\theta$ for $n=3$ and $n=10$, respectively. The angular functions of the normalized stresses closely resemble HRR asymptotic solutions (e.g. see the solutions plotted in PAN, 1990). The solutions for this case serve as a check for the accuracy of our finite element program.

\section{Plastic zones}

Figure 7 ( $a$ and b) shows the plastic zone size and shape for Cases 1-5 in the normalized coordinates $\bar{x}\left(=x \sigma_{0}^{2} / J E\right)$ and $\bar{y}\left(=y \sigma_{0}^{2} / J E\right)$ for $n=3$ and $n=10$, 
(c)


Fic. 6c and d.

respectively. The results of the plastic zone sizes and shapes for the pure mode I case $\left(M_{13}=1\right)$ agree with the results of SHIH $(1973,1974)$. The plastic zone sizes and shapes for $M_{13}^{e}=1$ and 0.83 and for $M_{13}^{c}=0$ and 0.17 are close to each other. As shown in the figures, when the mode III contribution increases, the plastic zone size increases. Also, as the hardening exponent $n$ increases, the plastic zone shifts to the front of the tip. This trend agrees well with those of the pure mode I and the pure mode III cases.

\section{CONCLUSIONS}

We have obtained numerical solutions for a crack in a power-law hardening material under small-scale yielding conditions and for a rather complete range of loadings 
(a)



(b)

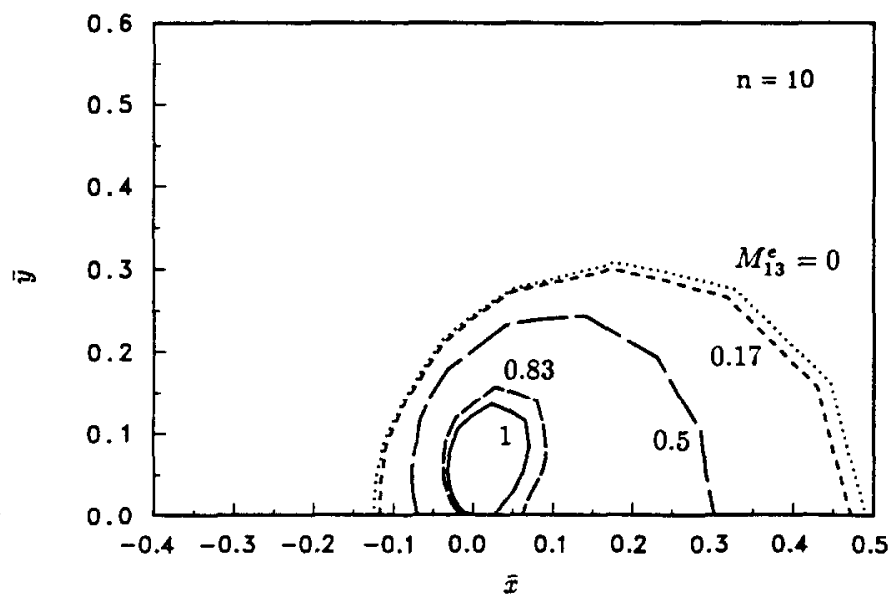

FIG. 7. The plastic zones for Cases $1-5$ plotted in the normalized coordinates $\bar{x}\left(=x \sigma_{0}^{2} / J E\right)$ and $\bar{y}$ $\left(=y \sigma_{0}^{2} / J E\right)$ for (a) $n=3$ and (b) $n=10$.

between pure mode I and pure mode III. Our computational results show that within the plastic zone, the in-plane stresses are more singular than the out-of-plane shear stresses. The qualitative nature and quantitative results for the angular functions and the singularity differences of either the in-plane stresses or the out-of-plane shear stresses from the HRR singularity under either large $M_{13}^{p}$ or small $M_{13}^{p}$ conditions agree well with the results from the perturbation analysis of PAN (1990).

Within the plastic zone, the plots of the normalized in-plane stresses or the normalized out-of-plane shear stresses as functions of $r / r_{p}$ in a $\log -\log$ scale in Figs 3-5 resemble straight lines when one or the other is more dominant. But when the contributions of the in-plane stresses and the out-of-plane shear stresses to the effective stress are comparable, these lines are curved. In the interval where the in-plane and 
out-of-plane stresses are comparable, the stresses cannot be expressed in separable forms of $r$ and $\theta$, as can those of the HRR fields under either pure in-plane or pure out-of-plane shear loading conditions.

In this paper, we have investigated the stress fields at distances ranging from $r / r_{p}=1$ to $r / r_{p} \approx 10^{-13}$ to $10^{-14}$. For typical structural steels, finite deformation effects should be taken into consideration at radial distances to the crack tip smaller than $r / r_{p} \approx 10^{-3}$. $\Lambda$ s mentioned previously, we investigated fields over distances that are well below those that are physically relevant in order to study the mathematical structure of the plastic near-tip fields. Of course, in applying these solutions to the analysis of fracture under combined-mode loading, fields within such physically irrelevant distances would be disregarded.

Within the small-scale yielding formulation, our numerical solutions suggest that the out-of-plane shear stresses are less singular than the in-plane stresses. Thus, the out-of-plane shear stresses (relative to the in-plane stresses) become vanishingly small as $r / r_{p} \rightarrow 0$. Nevertheless, the out-of-plane shear stresses may not be negligible over length scales that are physically relevant; this can be seen in Figs 3-5. An estimate can be made of the magnitude of the stresses at a microstructurally relevant radial distance from the crack front using the results presented here and in the perturbation analysis of PAN (1990). Finally, under combined mode I and III loadings and smallscale yielding conditions, it appears that linear elastic fracture mechanics analysis may possibly overestimate the effect of the near-tip out-of-plane shear stresses and underestimate the relative magnitude of the near-tip in-plane stresses.

\section{ACKNOWLEDGEMENT}

J.P. acknowledges the support of this work by the National Science Foundation under Grant No. MSM-8613544 at The University of Michigan. C.F.S. acknowledges the support of this work by the Material Research Group funded by the National Science Foundation at Brown University. Helpful discussions on this subject with Professor J. R. RICE of Harvard University are greatly appreciated.

\section{REFERENCES}

DONG, P. and PAN, J.

Dong, P. and PAN, $\mathbf{J}$.

Hughes, T. J. R. HutChinson, J. W. HUTCHINSON, J. W. KANNINEN, M. F. and Popelar, C. H. 1989a Plane-strain mixed-mode near-tip fields in elastic perfectly plastic solids under small-scale yielding conditions, submitted for publication in Int. J. Fracture.

1989 b Asymptotic crack-tip fields for perfectly plastic solids under plane-stress and mixed-mode loading conditions, submitted for publication in J. appl. Mech.

1980 Int. J. Numer. Meth. Engng 9, 187.

1968 a J. Mech. Phys. Solids 16, 13.

1968b J. Mech. Phys. Solids 16, 337.

1985 Adianced Fracture Mechanics, Oxford University Press. 
LI, F. Z. and PAN, J.

LI, F. Z. and PAN, J.

LI, F. Z. SHIH, C. F. and NeEdleman, $A$.

Miglin, M. T., HiRTh, J. P. and ROSENFIELDS, A. R.

Moran, B. and Shih, C. F.

MaLkuS, D. S. and HuGHes, T. J. R.

NagtegaAl, J. C., Parks, D. M. 1974 and RICE, J. R.

Nemat-Nasser, S. and Obata, M. 1984

PAN, J.

PAN, J. and SHIH, C. F.

PAN, J. and SHIH, C. F.

RICE, J. R.

RICE, J. R.

Rice, J. R. and Rosengren, G. F. 1968

SAKA, M., ABE, H. and TANAKA, $S$.

SHIH, C. F.

1990

1986

1988

$1968 \mathrm{a}$

$1968 \mathrm{~b}$

1986

1973

SHIH, C. F.

1974

ShiH, C. F. and Needleman, A.

Shih, C. F., Moran, B. and NaKamura, $T$.
Plane-strain crack-tip fields for pressure-sensitive dilatant materials, to appear in $J$. appl. $\mathrm{Mech}$. Plane-stress crack-tip fields for pressure-sensitive dilatant materials, to appear in Engng Fracture Mech.

Engng Fracture Mech. 21, 405.

Res. Mechanica 11, 85 .

Engng Fracture Mech. 27, 615.

Comput. Meth. Appl. Mech. Engng 15, 63.

Comput. Meth. Appl. Mech. Engng 4, 153.

Mech. Mater. 3, 235.

J. Mech. Phys. Solids 38, 133.

Mech. Mater. 5, 299.

Int. J. Fracture 37, 171.

J. appl. Mech. 35, 379.

In Fracture, Vol. 2, pp. 191-311 (edited by $\mathrm{H}$. Liebowitz), Academic Press, New York, NY. J. Mech. Phys. Solids 16, 1.

Comput. Mech. 1, 11.

Elastic-plastic analysis of combined mode crack problems, Ph.D. Thesis, Harvard University, Cambridge, MA.

Fracture Anal. ASTM STP 560, 187.

1984 J. appl. Mech. 51, 48.

1986 Int. J. Fracture 30, 79. 